\title{
Un varón con lesiones mucosas como signo de una enfermedad oculta (pénfigo paraneoplásico)
}

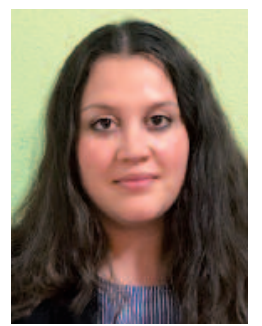

\section{Marta Prieto-Barrios}

Médico residente de $4 .^{\circ}$ año de Dermatología.

Hospital Universitario

12 de Octubre. Madrid.
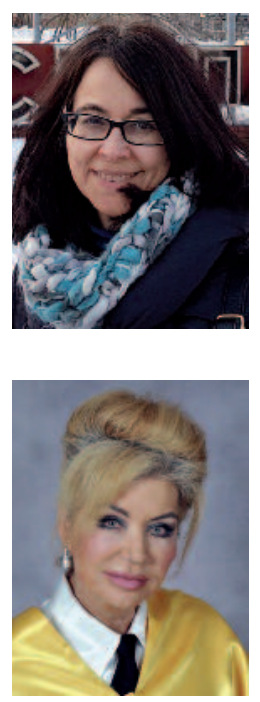

Beatriz García-Bracamonte

Facultativo especialista de área. Servicio de Dermatología. Hospital Universitario 12 de Octubre. Madrid.

\section{Aurora Guerra-Tapia}

Jefa de la Sección de Dermatología. Hospital Universitario 12 de Octubre. Madrid. Profesora titular de Dermatología Médico-Quirúrgica y Venereología.

Universidad Complutense de Madrid.

Profesora de Dermatología. Universidad Internacional de La Rioja (UNIR).
Mi paciente es un varón de 66 años, fumador, sin otros antecedentes de interés, que comenzó a presentar lesiones en la lengua, que le producían escasas molestias subjetivas. Acudió a su médico, quien sugirió que podrían ser hongos y le pautó un antifúngico tópico. Poco tiempo después, comenzó con enrojecimiento y constante lagrimeo en ambos ojos. De nuevo, consultó por estos síntomas, que se interpretaron en aquella ocasión como una posible conjuntivitis bacteriana bilateral.

Sin embargo, cuatro días después, el paciente, que continuaba empeorando, detectó la presencia de lesiones eritematosas tanto en las palmas como las en plantas, y acudió a nuestro servicio de urgencias de dermatología.

El paciente estaba muy sintomático, con dificultades para comer, y las pautas tópicas que había recibido no parecían hacerle mejorar. Las lesiones orales, de aspecto pseudomembranoso, se habían extendido a toda la lengua, la mucosa yugal y la cara interna de los labios, mostrando erosiones y costras en hemimucosas. La conjuntiva ocular estaba eritematosa y presentaba importante exudado espeso amarillento. En las palmas y plantas, mostraba lesiones eritematoedematosas papulosas salpicadas y algunas de ellas adquirían morfología en diana (fig. 1).

Sospechando un cuadro del tipo eritema exudativo multiforme mayor o síndrome de Stevens-Johnson, se realizó una anamnesis dirigida a las posibles causas que lo pudieran haber provocado.

El paciente negaba haber tomado en los días previos ningún tipo de fármaco o producto de herbolario y tampoco realizaba ningún tratamiento habitual. No refería haber sufrido episodios de herpes labial o genital. No tenía fiebre y, salvo un leve aumento de 

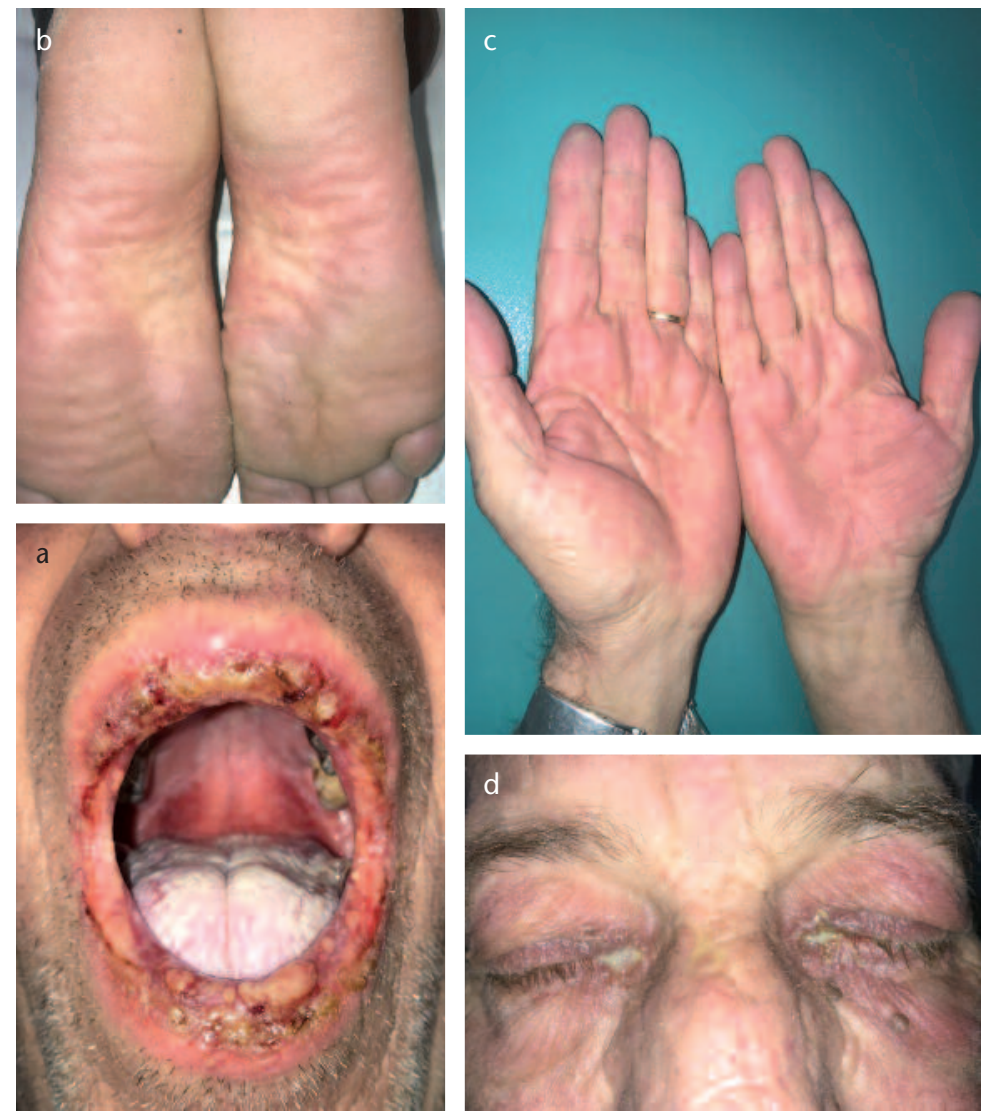

Figura 1. a) Lesiones erosivas en la mucosa oral. b) y c) Lesiones eritematoedematosas, algunas de morfología en diana, que afectan a las palmas y plantas. d) Exudado amarillento conjuntival bilateral.

tos en los últimos días, no se había notado otra clínica sistémica.

La analítica de sangre reveló únicamente un aumento de la proteína C-reactiva de $5,69 \mathrm{mg} / \mathrm{dL}$ (intervalo normal: $0,10-0,50 \mathrm{mg} / \mathrm{dL}$ ).

Con todos estos datos, ante la ausencia de pistas sobre el posible desencadenante de esta reacción inflamatoria, se decidió solicitar una placa de tórax urgente para descartar la existencia de una infección respiratoria por Mycoplasma pneumoniae, una de las posibles causas más frecuentes.

Cuál fue la sorpresa cuando la imagen de esta radiografía reveló la existencia de una masa de varios centímetros de diámetro, con bordes irregulares, en el lóbulo inferior izquierdo (fig. 2).

El paciente ingresó en planta de dermatología, donde se inició el tratamiento con corticoides sis- témicos y se realizaron las pruebas para completar el estudio.

Se efectuaron dos biopsias en fresco: una de la palma de la mano derecha y otra de la mucosa labial. Mientras que la primera fue inespecífica, la segunda mostraba una mucosa oral con una solución de continuidad y sustitución del epitelio por depósitos de fibrina, presentando en la periferia una ampolla intraepidérmica con acantólisis, además de infiltrado linfocitario en banda con aislados eosinófilos. Con las técnicas de inmunofluorescencia directa, se detectaron depósitos lineales basales de inmunoglobulina $\mathrm{G}$ y fracción 3 del complemento. La inmunofluorescencia indirecta fue negativa para las desmogleínas y los anticuerpos anti-membrana basal.

Se le hizo también una tomografía computarizada, que aportó una imagen de mayor resolución de la masa tumoral de $5 \mathrm{~cm}$ en el lóbulo inferior izquierdo, altamente sugestiva de malignidad.

Todo esto nos permitió realizar el diagnóstico de pénfigo paraneoplásico.

El pénfigo es una enfermedad ampollosa autoinmunitaria poco frecuente, que se caracteriza por la existencia de anticuerpos que atacan la superficie de las células de la piel y las mucosas y provocan la pérdida de la unión entre ellas ${ }^{1}$. Existen diferentes tipos, siendo los más frecuentes el pénfigo vulgar, que se asocia a la antidesmogleína 3, y el pénfigo foliáceo, que tiene antidesmogleína 1.

El pénfigo paraneoplásico es un subtipo que se relaciona con la existencia de neoplasias subyacentes. Los tumores más frecuentemente asociados a este cuadro son los linfomas no hodgkinianos, la leucemia linfocítica crónica, los sarcomas y la macroglobulinemia de Waldenström. La enfer- 


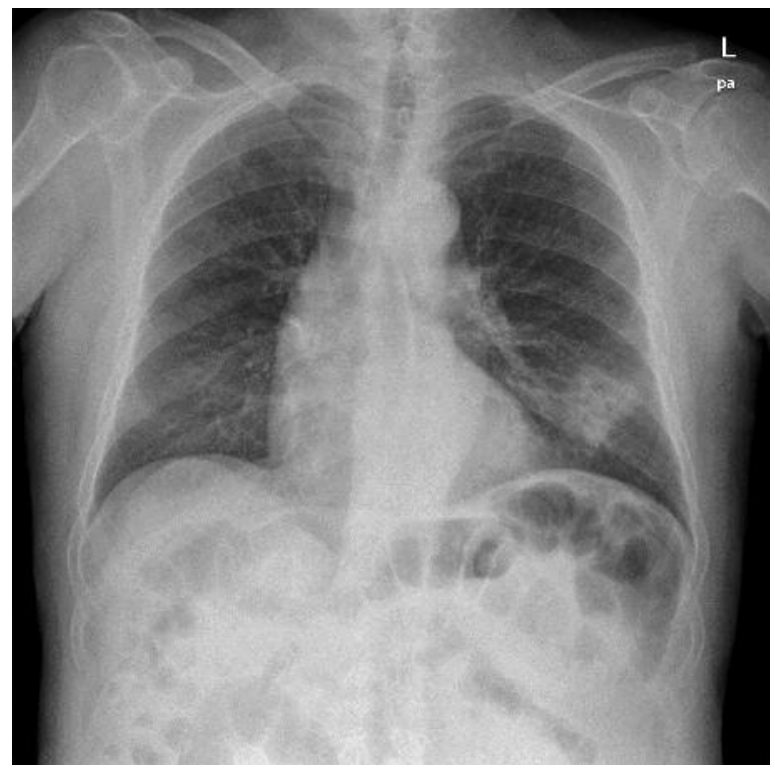

Figura 2. Radiografía de tórax que muestra una masa de bordes irregulares en el lóbulo inferior izquierdo.

medad de Castleman, que supone el $10 \%$ de los casos, es la que más se relaciona con las formas infantiles. También se han descrito variantes secundarias a tumores epiteliales, representando los carcinomas un $8,6 \%$ de los casos $^{2}$.

Cuando Anhalt et al. describieron este síndrome en $1990^{3}$, establecieron unos criterios diagnósticos, que, posteriormente, fueron revisados por Helm et al. y que se exponen en la tabla $1^{4}$. Estos criterios siguen estando en vigor. Sin embargo, la prueba diagnóstica más específica sería la detección de anticuerpos antiplaquina en suero, que, a día de hoy, es difícil de realizar en la práctica clínica ${ }^{5}$.

La principal característica del pénfigo paraneoplásico es la afectación mucosa predominante e importante, con una estomatitis dolorosa, y lesiones cutáneas polimorfas, que, en ocasiones, remedan el eritema exudativo multiforme. La conjuntivitis pseudomembranosa también es habitual y puede dar lugar a cicatrices. Se ha descrito, asimismo, afectación esofágica, nasofaríngea y de la mucosa genital, y puede desarrollarse una bronquiolitis obliterante grave, que puede resul-
Tabla 1. Criterios diagnósticos del pénfigo paraneoplásico

\section{CRITERIOS MAYORES}

1. Erupción mucocutánea polimorfa.

2. Neoplasia interna concomitante.

3. Hallazgos de inmunoprecipitación característicos del suero.

\section{CRITERIOS MENORES}

1. Evidencia histológica de acantólisis.

2. Tinción del epitelio transicional de la vejiga de rata mediante inmunofluorescencia indirecta.

3. Depósitos de inmunorreactantes en los espacios intercelulares y la membrana basal mediante inmunofluorescencia directa de la piel perilesional.

Son necesarios tres criterios mayores o dos mayores y dos menores para realizar el diagnóstico.

tar mortal ${ }^{6}$. Los hallazgos histológicos, al igual que los clínicos, son variados y muestran acantólisis con alteraciones liquenoides o de interfase ${ }^{7}$.

El manejo de estos casos es complicado y la enfermedad tiende a ser recalcitrante. La mayoría de los procesos precisa inmunosupresión sistémica mantenida y es necesario el tratamiento de la enfermedad de base $^{8}$. La afectación oral, que suele ser el signo inicial más temprano, también suele ser la más resistente al tratamiento.

Desafortunadamente, nuestro paciente, durante el ingreso, presentó un dolor precordial como manifestación de un síndrome coronario sin elevación del segmento ST y precisó ser sometido a un procedimiento de revascularización. Esto ha dado lugar a un retraso en las pruebas diagnósticas invasivas de la lesión pulmonar y el inicio de su tratamiento.

Consideramos que este caso sirve como recordatorio de este cuadro autoinmunitario infrecuente que puede ocurrir en pacientes con neoplasias ocultas. Es importante tener en cuenta que, ante lesiones mucosas en distintas localizaciones, como la oral, la conjuntiva de ambos ojos o la región 
genital, se debe sospechar como primera posibilidad la existencia de un cuadro inflamatorio sistémico, antes que la coincidencia de procesos infecciosos concomitantes. Los dermatólogos desempeñamos un papel privilegiado, ya que, mediante una exploración clínica exhaustiva, incluso desde el departamento de urgencias, somos capaces de detectar enfermedades graves, que, de otra manera, podrían haber pasado desapercibidas.

\section{BIBLIOGRAFÍA}

1. Kasperkiewicz M, Ellebrecht CT, Takahashi H, Yamagami J, Zillikens D, Payne AS, et al. Pemphigus. Nat Rev Dis Primers. 2017;3:17026.

2. Sehgal VN, Srivastava G. Paraneoplastic pemphigus/paraneoplastic autoimmune multiorgan syndrome. Int J Dermatol. 2009;48(2):162-9.
3. Anhalt GJ, Kim SC, Stanley JR, Korman NJ, Jabs DA, Kory M, et al. Paraneoplastic pemphigus. An autoimmune mucocutaneous disease associated with neoplasia. N Engl J Med. 1990;323(25):1729-35.

4. Helm TN, Camisa C, Valenzuela R, Allen CM. Paraneoplastic pemphigus. A distinct autoimmune vesiculobullous disorder associated with neoplasia. Oral Surg Oral Med Oral Pathol. 1993;75(2):209-13.

5. Hashimoto T, Amagai M, Watanabe K, Chorzelski TP, Bhogal BS, Black MM, et al. Characterization of paraneoplastic pemphigus autoantigens by immunoblot analysis. J Invest Dermatol. 1995;104(5):829-34.

6. Mascaró Galy JM, Iranzo Fernández P, Herrero Mateu C. Pénfigo paraneoplásico. Piel. 2007;22(2):63-71.

7. Díaz Martínez MA, Almodóvar Real AM, Sánchez López J, Navarro Triviño FJ, Naranjo Sintes R, Ruiz Villaverde R. Pénfigo paraneoplásico y cáncer de pulmón asociados a miastenia gravis. Piel. 2016;31(2):92-5.

8. Ruocco E, Wolf R, Ruocco V, Brunetti G, Romano F, Lo Schiavo A. Pemphigus: associations and management guidelines: facts and controversies. Clin Dermatol. 2013;31(4):382-90. 